\title{
ON ANALYTIC DIAMETERS AND ANALYTIC CENTERS OF COMPACT SETS
}

BY

\author{
SHŌJI KOBAYASHI AND NOBUYUKI SUITA
}

\begin{abstract}
In this paper several results on analytic diameters and analytic centers are obtained. We show that the extremal function for analytic diameter is unique and that there exist compact sets with many analytic centers. We answer negatively several problems posed by F. Miinsker.
\end{abstract}

Introduction. In the theory of functions in the complex plane, many capacitytype set functions are defined in order to measure the size of sets. The notions of analytic diameter and analytic center were first introduced by Vitushkin [13], [14] in the way of developing his beautiful theory of rational approximation. Recently F. Minsker [9] obtained their several properties and posed in the last part of his paper [9, p. 93] open problems on them. In this paper we investigate certain properties of the extremal function for the problem of analytic diameters and construct instructive examples, most of which offer negative answers to Minsker's problems.

In $\S 1$ we list the definitions and the notation which we use throughout this paper. In $\$ 2$ we state preliminary known results as a series of lemmas. In $\$ 3$ we derive a property of the extremal function, and as a corollary we show that the extremal function is unique. In $\$ 4$ we consider the case where the compact set consists of finitely many continua. In $\$ 5$ we give examples, most of which serve as counterexamples to Minsker's problems.

1. Definition and notation. Let $K$ be a compact set in the complex plane, and let $D=D(K)$ denote the unbounded component of the complement $K^{c}$ of $K$ with respect to the Riemann sphere. By $A B(D)$ we denote the Banach space of all bounded analytic functions in $D$, normed by the uniform norm $\|\cdot\|_{\infty}$ in $D$, that is

$$
\|f\|_{\infty}=\sup \{|f(z)|: z \in D\}
$$

for any $f \in A B(D)$. Let

$$
\mathcal{Q}(K)=\left\{f \in A B(D):\|f\|_{\infty} \leqslant 1, f(\infty)=0\right\} .
$$

Any function which belongs to $\mathscr{Q}(K)$ is of ten called an admissible function for $K$.

The analytic capacity $\gamma(K)$ of $K$ is defined by

$$
\gamma(K)=\sup \left\{\left|f^{\prime}(\infty)\right|: f \in \mathbb{Q}(K)\right\} .
$$

It is well known that there exists a unique extremal function $f_{0} \in \mathbb{Q}(K)$ with $f_{0}^{\prime}(\infty)=\gamma(K)$, which is called the Ahlfors function of $K[1]-[4]$, [7], [8]. 
Any function $f$ analytic at $\infty$ can be expressed in the form

$$
f(z)=f(\infty)+f^{\prime}(\infty) /\left(z-z_{0}\right)+\beta\left(f, z_{0}\right) /\left(z-z_{0}\right)^{2}+\ldots,
$$

for an arbitrary complex number $z_{0}$. Here $\beta\left(f, z_{0}\right)$ is given by

$$
\beta\left(f, z_{0}\right)=\frac{1}{2 \pi i} \int_{|z|=R} f(z)\left(z-z_{0}\right) d z
$$

for sufficiently large $R>0$. A simple calculation shows

$$
\beta\left(f, z_{1}\right)=\beta\left(f, z_{0}\right)+\left(z_{0}-z_{1}\right) f^{\prime}(\infty)
$$

for arbitrary complex numbers $z_{0}$ and $z_{1}$.

For each compact set $K$ with $\gamma(K)>0$, define

$$
\beta(K, z)=\sup \{|\beta(f, z)| / \gamma(K): f \in \mathbb{Q}(K)\} .
$$

Using (5), we easily see that

$$
\left|\beta\left(K, z_{0}\right)-\beta\left(K, z_{1}\right)\right| \leqslant\left|z_{0}-z_{1}\right|,
$$

in particular, $\beta(K, z)$ is a continuous function of $z$, and that

$$
\lim _{z \rightarrow \infty} \beta(K, z)=+\infty
$$

(see [5], [9]). If $\gamma(K)=0$ define $\beta(K, z)=0$ for every complex number $z$. The analytic diameter $\beta(K)$ of $K$ is defined by

$$
\beta(K)=\inf _{z} \beta(K, z) .
$$

Any point $w$ for which $\beta(K)=\beta(K, w)$ is called the analytic center of $K$, and we denote by $c(K)$ the set of all analytic centers of $K$. From (7) and (8), we easily see that $c(K)$ is a nonempty compact set.

It is well known that if $\gamma(K)>0$, then for every complex number $z$ there exists a unique function $f \in \mathbb{Q}(K)$ which takes the supremum in (6), that is,

$$
\beta(K, z)=\beta(f, z) / \gamma(K)
$$

[3], [4], [8]. We call such a function the $z$-extremal function and denote it by $f_{z}$. Any $z$-extremal function with $z \in c(K)$ is called the $\beta$-extremal function. In $\$ 3$ it is proved that the $\beta$-extremal function is unique while $c(K)$ can contain more than one point. The $\beta$-extremal function is denoted by $f_{1}$.

Since all concepts defined above remain unchanged if we add to $K$ the relatively compact components of $K^{c}$, throughout this paper we assume that $K^{c}$ has no relatively compact components.

2. Preliminary lemmas. In this section we state several results on analytic diameters and analytic centers as a series of lemmas. Lemma 1 states their dependence on conformal maps.

Lemma 1. Let $K_{j}(j=1,2)$ be two compact sets. Suppose that there exists a conformal map $\phi$ of $D\left(K_{1}\right)$ onto $D\left(K_{2}\right)$ with an expansion

$$
\phi(z)=z+a_{0}+a_{1} z^{-1}+\ldots
$$

about $\infty$; then

$$
\beta\left(K_{1}\right)=\beta\left(K_{2}\right)
$$


and

$$
c\left(K_{1}\right)+a_{0}=c\left(K_{2}\right),
$$

where by $c\left(K_{1}\right)+a_{0}$ we denote the set $\left\{w+a_{0}: w \in c\left(K_{1}\right)\right\}$.

Proof. If $f \in \mathbb{Q}\left(K_{2}\right)$, then $g=f \circ \phi \in \mathbb{Q}\left(K_{1}\right)$. A simple calculation shows

$$
\beta(g, z)=\beta\left(f, z-a_{0}\right),
$$

from which (12) and (13) follow.

LEMMA 2. $f_{z}$ converges to $f_{w}$ on every compact subset of $D(K)$ as $z \rightarrow w$.

Proof. The lemma follows from a routine normal family argument.

LEMMA 3. $\beta(K) \geqslant \gamma(K)$.

For the proof see $[5$, p. 209] or $[9$, p. 85].

LEMMA 4. If $K$ is symmetric with respect to a line, then so are $\beta(K, z)$ and $c(K)$.

LEMMA 5. If $K$ is a continuum which contains more than one point, then

$$
\beta(K)=\gamma(K)
$$

and $c(K)$ is a singleton which is contained in the convex hull of $K$.

For the proofs of Lemmas 4 and 5 see [9, pp. 87-88].

3. Uniqueness of the $\beta$-extremal function. In this section we prove several results which hold for general compact sets.

THEOREM 1. Suppose that $f_{1}$ is a $\beta$-extremal function; then $f_{1}^{\prime}(\infty)=0$.

Proof. By definition we see that $f_{1}=f_{z}$ for some $z \in c(K)$. Using (5), (6) and (9), we have

$$
\gamma(K) \beta(K)=\frac{1}{2} f_{z}^{\prime \prime}(\infty)-z f_{z}^{\prime}(\infty) \leqslant \frac{1}{2} f_{w}^{\prime \prime}(\infty)-w f_{w}^{\prime}(\infty)=\gamma(K) \beta(K, w)
$$

and

$$
\left|\beta\left(f_{w}, z\right)\right|=\left|\frac{1}{2} f_{w}^{\prime \prime}(\infty)-z f_{w}^{\prime}(\infty)\right| \leqslant \gamma(K) \beta(K)
$$

for every complex number $w$. Therefore we see

$$
\left|f_{w}^{\prime \prime}(\infty)-z f_{w}^{\prime}(\infty)\right| \leqslant \frac{1}{2} f_{w}^{\prime \prime}(\infty)-w f_{w}^{\prime}(\infty)
$$

Comparing the real part of (18), we obtain

$$
\operatorname{Re}(w-z) f_{w}^{\prime}(\infty) \leqslant 0 .
$$

Write $w-z=r e^{i \theta}$; then (19) becomes

$$
\operatorname{Re} e^{i \theta} f_{w}^{\prime}(\infty)<0 .
$$

By letting $r \rightarrow 0$, we obtain

$$
\operatorname{Re} e^{i \theta} f_{z}^{\prime}(\infty)<0,
$$

since $f_{w}^{\prime}(\infty) \rightarrow f_{z}^{\prime}(\infty)$ as $w \rightarrow z$ by Lemma 2 . Since (21) holds for every real number $\theta$, we see $f_{z}^{\prime}(\infty)=0$ as desired. 
COROLlary. There exists a unique $\beta$-extremal function.

Proof. Let $z, w \in c(K)$; all we must show is $f_{z}=f_{w}$. By the theorem, we see

$$
f_{z}^{\prime}(\infty)=f_{w}^{\prime}(\infty)=0 \text {. }
$$

By (6) and (9) we see

$$
\begin{aligned}
\gamma(K) \beta(K) & =\gamma(K) \beta(K, z)=\frac{1}{2} f_{z}^{\prime \prime}(\infty)-z f_{z}^{\prime}(\infty)=f_{z}^{\prime \prime}(\infty)=\frac{1}{2} f_{z}^{\prime \prime}(\infty)-w f_{z}^{\prime}(\infty) \\
& \leqslant \frac{1}{2} f_{w}^{\prime \prime}(\infty)-w f_{w}^{\prime}(\infty)=\gamma(K) \beta(K, w)=\gamma(K) \beta(K) .
\end{aligned}
$$

Therefore we obtain

$$
\beta\left(f_{w}, z\right)=\gamma(K) \beta(K, z),
$$

which means that $f_{w}$ is the $z$-extremal function, i.e. $f_{z}=f_{w}$.

Next we give a rough estimate of the diameter of $c(K)$, which may be improved to a certain degree.

Theorem 2. diam $c(K) \leqslant 2 \beta(K)$.

Proof. Let $z_{j} \in c(K)$ for $j=1,2$, and $f_{0}$ be the Ahlfors function; then using (6) we see

$$
\left|\beta\left(f_{0}, z_{j}\right)\right| \leqslant \gamma(K) \beta\left(K, z_{j}\right)=\gamma(K) \beta(K)
$$

By (5), we obtain

$$
\left|z_{1}-z_{2}\right| \leqslant 2 \beta(K)
$$

since $f_{0}^{\prime}(\infty)=\gamma(K)$ by $(2)$.

4. Finitely many continua. In this section we consider the case where $K$ consists of a finite number of mutually disjoint continua each of which contains more than one point.

Making use of auxiliary conformal maps together with Lemma 1, if necessary, we may assume that the boundary $\partial K$ of $K$ consists of mutually disjoint analytic Jordan curves. Let $m$ denote the connectivity of $D(K)$, which coincides with the number of the continua since we assumed that $K^{c}$ has no relatively compact components. Throughout this section we assume that $D(K)$ is a domain bounded by $m$ mutually disjoint analytic Jordan curves.

If $h$ is a meromorphic function or differential on $\bar{D}$, then by $Z(h)$ and $P(h)$ we denote the number of zeros and poles, respectively, on $\bar{D}$ counting multiplicities, except that those on $\partial D$ are counted according to half their multiplicities. The following theorem corresponds to Corollary 9.6 of [6].

THEOREM 3. Let $f_{1}$ be the $\beta$-extremal function; then

$$
m \leqslant Z\left(f_{1}\right) \leqslant m+1 .
$$

Proof. Let $z_{0} \in c(K)$. By Cauchy's formula we see for any $f \in \mathbb{Q}(K)$,

$$
\beta\left(f, z_{0}\right)=\frac{1}{2 \pi i} \int_{\partial K}\left(z-z_{0}\right) f(z) d z .
$$


Using the Hahn-Banach theorem and Riesz's representation theorem (see, for example, [12, p. 138]), we see that there exists a complex measure $\mu$ on $\partial K$ such that

$$
\beta\left(f, z_{0}\right)=\int_{\partial K} f(z) d \mu(z)
$$

for any $f \in \mathcal{Q}(K)$, and that

$$
\|\mu\|=\gamma(K) \beta\left(f_{1}, z_{0}\right)=\gamma(K) \beta(K) .
$$

By (27) and (28) we see

$$
\int_{\partial K} f(z)\left(\frac{1}{2 \pi i}\left(z-z_{0}\right) d z-d \mu(z)\right)=0
$$

for every $f \in \mathbb{Q}(K)$. Therefore, by F. and M. Riesz's theorem (see, for example, [6] or [11]), there is a function $g \in H^{1}(D)$ with $g(\infty)=0$ such that

$$
(2 \pi i)^{-1}\left(z-z_{0}\right) d z-d \mu(z)=(2 \pi i)^{-1} g(z) d z
$$

along $\partial K$, where $H^{1}(D)$ denotes the Hardy class, the class of analytic functions $h$ for which $|h|$ admits a harmonic majorant in $D$. Then

$$
\beta\left(f, z_{0}\right)=\frac{1}{2 \pi i} \int_{\partial K} f(z)\left(z-z_{0}-g(z)\right) d z,
$$

for every $f \in \mathbb{Q}(K)$. By (29) we see

$$
\begin{aligned}
\frac{1}{2 \pi} \int_{\partial K}\left|z-z_{0}-g(z)\right||d z| & =\gamma(K) \beta(K) \\
& =\frac{1}{2 \pi i} \int_{\partial K} f_{1}(z)\left(z-z_{0}-g(z)\right) d z
\end{aligned}
$$

and hence

$$
(1 / i) f_{1}(z)\left(z-z_{0}-g(z)\right) d z \geqslant 0
$$

almost everywhere along $\partial K$, and

$$
\left|f_{1}(z)\right|=1
$$

almost everywhere on $\partial K$. Therefore it is seen that $f_{1}$ and $g$ are both analytically continued across $\partial K$ and (34) and (35) hold everywhere (see [11, p. 60]). Using the argument principle, we see by (35),

$$
Z\left(f_{1}\right) \geqslant m
$$

and by (34),

$$
Z\left(f_{1}\left(z-z_{0}-g\right) d z\right)-P\left(f_{1}\left(z-z_{0}-g\right) d z\right)=m-2 .
$$

Therefore we see

$$
Z\left(f_{1}\right) \leqslant m+1,
$$

since $P\left(f_{1}\left(z-z_{0}-g\right) d z\right)=1$. Combining (36) and (38), we have proved the theorem.

THEOREM 4. $c(K)$ contains more than one point if and only if there exists a nonconstant analytic differential $\omega$ on $\bar{D}$ which is real along $\partial K$ and which has the same zeros in $D-\{\infty\}$ as $f_{1}$ with multiplicities taken into account. 
Proof. Suppose that $c(K)$ contains more than one point. Let $z_{j}(j=1,2)$ be two different analytic centers of $K$ and $g_{j}$ be a corresponding $H^{1}$ function, respectively, which we defined in the proof of Theorem 3. Define

$$
\omega=(1 / i) f_{1}(z)\left(z_{1}-z_{2}-g_{1}(z)+g_{2}(z)\right) d z .
$$

Then, by using (34), we easily see that $\omega$ satisfies the required conditions.

For the converse suppose that there exists such an $\omega$ as mentioned in the theorem. Let $z_{0} \in c(K)$ and $g$ be a corresponding $H^{1}$ function as above. Set

$$
\alpha=(1 / i) f_{1}(z)\left(z-z_{0}-g(z)\right) d z .
$$

Then $\alpha$ is a meromorphic differential on $\bar{D}$ which is nonnegative along $\partial K$. By (37) we see

$$
Z(\alpha)=m-1 \text {. }
$$

Since $f_{1}$ has no zeroes on $\partial K$ by (35), we see from (34) and (41) that $\alpha$ has at most one double zero on $\partial K$. If $\alpha$ has a double zero on $\partial K$, let $\Gamma_{k}$ be the component of $\partial K$ on which the zero lies. Then $\alpha$ is nonnegative along $\Gamma_{k}$ and positive along $\partial K-\Gamma_{k}$. Since $Z(\omega)=m-2, \omega$ has no zeros on $\partial K$. If necessary, by considering $-\omega$ we may assume that $\omega$ is positive along $\Gamma_{k}$. If $\alpha$ has no zeros on $\partial K$, then $\alpha$ is positive along $\partial K$. Therefore, in both cases, we see that for a sufficiently small positive number $\varepsilon$

$$
\alpha+\varepsilon \omega>0
$$

along $\partial K$. Since $\omega$ has no zero at $\infty, \omega$ is expanded about $\infty$ as follows:

$$
\omega=\left(A z^{-2}+\ldots\right) d z
$$

where $A \neq 0$. Then, for any $f \in \mathcal{Q}(K)$, we see by Cauchy's formula:

$$
\begin{aligned}
& \frac{1}{2 \pi i} \int_{\partial K} f(z)\left(\left(z-z_{0}-g(z)\right) d z+\frac{\varepsilon \omega}{f_{1}(z)}\right) \\
& \quad=\frac{1}{2} f^{\prime \prime}(\infty)-z_{0} f^{\prime}(\infty)+\frac{\varepsilon A}{\gamma(K) \beta(K)} f^{\prime}(\infty)=\beta\left(f, z_{0} \frac{\varepsilon A}{\gamma(K) \beta(K)}\right) .
\end{aligned}
$$

Set $z_{1}=z_{0}-\varepsilon A / \gamma(K) \beta(K)$. Then we see by (42) and (44),

$$
\begin{aligned}
\left|\beta\left(f, z_{1}\right)\right| & \leqslant \frac{1}{2 \pi} \int_{\partial K}\left|\left(z-z_{0}-g(z)\right) d z+\frac{\varepsilon \omega}{f_{1}(z)}\right| \\
& =\frac{1}{2 \pi} \int_{\partial K} f_{1}(z)\left(z-z_{0}-g(z)\right) d z+\varepsilon \omega=\frac{1}{2} f_{1}^{\prime \prime}(\infty) .
\end{aligned}
$$

This means $f_{1}$ is the $z_{1}$-extremal function, i.e. $z_{1} \in c(K)$.

COROllary. If $Z\left(f_{1}\right)=m+1$, then $K$ has the unique analytic center.

Proof. Suppose that $c(K)$ contains more than one point. Then by the theorem there exists a nonconstant analytic differential $\omega$ on $\bar{D}$ which is real along $\partial D$ and which has the same zeros as $f_{1}$ in $D-\{\infty\}$. In particular, we see $Z(\omega)=m-1$. But it is well known that any analytic differential $\omega$ on $\bar{D}$ which is real along $\partial K$ satisfies $Z(\omega)=m-2$. This is a contradiction. 
THEOREM 5. $c(K)$ is either a singleton or a line segment.

Proof. By the corollary to Theorem 4, we may assume that $Z\left(f_{1}\right)=m$. Let $z_{j}$ $(j=1,2) \in c(K)$ and $g_{j}$ be as in the proof of Theorem 4 . Let $\mathfrak{B}$ be the real linear space which consists of all analytic differentials on $\bar{D}$ which are real along $\partial K$ and which has the same zeros in $D-\{\infty\}$ as $f_{1}$ with multiplicities taken into account. If $\omega_{1}, \omega_{2} \in \mathfrak{B}$, then $\omega_{1} / \omega_{2}$ is constant in $D$, since it has no zeros. Therefore we see that $\mathfrak{B}$ is one dimensional, i.e.

$$
\mathscr{B}=\left\{t \omega_{0} ; t \in R\right\}
$$

for some $\omega_{0} \in \mathfrak{B}$. Write

$$
\omega_{0}=\left(A z^{-2}+\ldots\right) d z
$$

near $\infty$, where $A \neq 0$. Therefore we see

$$
\omega=(1 / i) f_{1}(z)\left(z_{1}-z_{2}-g_{1}(z)+g_{2}(z)\right) d z=t \omega_{0}
$$

for some real number $t$. Then we obtain

$$
z_{1}-z_{2}=i t A / \gamma(K) \beta(K),
$$

by comparing the coefficients. This means that $c(K)$ is either a singleton or a line segment.

THEOREM 6. If $m \geqslant 2$, then $\beta(K)>\gamma(K)$.

Proof. By Lemma 3, $\beta(K) \geqslant \gamma(K)$, so assume $\beta(K)=\gamma(K)$. Let $z \in c(K)$; then

$$
\gamma(K)=\beta(K)=\beta(z, K) .
$$

Let $F=f_{0}^{2}$, where $f_{0}$ is the Ahlfors function of $K$. Then we see

$$
\beta(F, z)=\gamma^{2}(K)=\gamma(K) \beta(K),
$$

which means that $F$ is the $\beta$-extremal function. Since $Z\left(f_{0}\right)=m$, we see that $Z(F)=2 m$, which contradicts Theorem 3 .

REMARK. Theorem 6 offers a negative answer to a problem presented by Minsker [9, Problem (1), p. 93].

5. Examples. In this section we give several examples which show that various cases considered in the previous section actually happen.

In Example 1, we give a method of evaluating the analytic diameter $\beta(K)$ when $K$ is a simple symmetric compact set on a line.

EXAMPLE $1[m=2]$. Let $0<a<b$ and $K=[-b,-a] \cup[a, b]$ be a symmetric compact set on the real line. Then $\beta(K)=(a+b) / 2$ and $c(K)=[-\delta, \delta]$ for some $\delta>0$.

Proof. Let $F_{0}$ be the Ahlfors function for the set $K^{2}=\left[a^{2}, b^{2}\right]$, and $f_{1}$ the $\beta$-extremal function of $K$. By Lemma 4 we see $0 \in c(K)$ and $f_{1}(z)=f_{1}(-z)$ for $z \in D(K)$. Therefore we can define a single-valued function $F_{1} \in \mathbb{Q}\left(K^{2}\right)$ such that

$$
F_{1}\left(z^{2}\right)=f_{1}(z) \quad(z \in D(K)) .
$$

By the extremal property of $F_{0}$, we see

$$
\left|F_{1}^{\prime}(\infty)\right| \leqslant F_{0}^{\prime}(\infty)=\gamma\left(K^{2}\right) .
$$


From (52) and the extremal property of $f_{1}$ we obtain

$$
F_{1}^{\prime}(\infty)=\beta\left(f_{1}, 0\right)=\gamma(K) \beta(K) .
$$

Combining (52) and (53), we see

$$
\gamma(K) \beta(K) \leqslant \gamma\left(K^{2}\right) .
$$

On the other hand let $g(z)=F_{0}\left(z^{2}\right)$ for $z \in D(K)$. Then $g \in \mathbb{Q}(K)$ and

$$
\beta(g, 0)=F_{0}^{\prime}(\infty)=\gamma\left(K^{2}\right) .
$$

From (54) and (55) we see that $g$ is the $\beta$-extremal function, i.e. $g=f_{1}$, and that

$$
\gamma(K) \beta(K)=\gamma\left(K^{2}\right) .
$$

By Pommerenke's theorem [10], we see that

$$
\gamma(K)=(b-a) / 2 \text { and } \gamma\left(K^{2}\right)=\left(b^{2}-a^{2}\right) / 4 .
$$

Hence, by (56), we obtain the desired result

$$
\beta(K)=(a+b) / 2 .
$$

Next we examine the set $c(K)$ of the analytic centers of $K$. By (52) we see that $f_{1}$ has no zeros in $D$ except the double zero at $\infty$. Therefore every analytic differential on $\bar{D}$ which is real along $\partial K$ satisfies the conditions mentioned in Theorem 4 . Since it is well known that such nonconstant differentials exist, we see that $c(K)$ is not a singleton by Theorem 4 . Moreover we easily see

$$
c(K)=[-\delta, \delta]
$$

for some $\delta>0$, since it is known by symmetry that every analytic differential which is real along $\partial K$ has a purely imaginary coefficient of $z^{-2} d z$ about $\infty$.

REMARK. Example 1 shows that $\beta$ is not monotone as a set function. This offers a negative answer to Minsker's problem [9, Problem (2), p. 93].

THEOREM 7. Let $K=K_{1} \cup K_{2}$, where the $K_{j}$ are disjoint continua for $j=1,2$. Let $\nu$ denote the harmonic measure of $D$ at $\infty$.

(a) If $\nu\left(K_{1}\right)=\frac{1}{2}$, then $Z(f)=2$ and $c(K)$ is a line segment (which is not a singleton).

(b) If $\nu\left(K_{1}\right) \neq \frac{1}{2}$, then $Z(f)=3$ and hence $c(K)$ is a singleton.

Proof. The first part of the theorem follows from Example 1, if necessary by using Lemma 1 . In order to prove the second part, suppose $Z\left(f_{1}\right)=2$, which means that $f_{1}$ has no zeros in $D$ except at $\infty$. Let $G(z, \infty)$ denote the Green's function of $D$ with pole at $\infty$. Set

$$
u(z)=\log \left|f_{1}(z)\right|+2 G(z, \infty) .
$$

Then $u$ is harmonic and bounded in $D$ and $u=0$ on $\partial K$, and hence $u(z)=0$ in $D$ by the maximum principle. Therefore we have

$$
f_{1}(z)=\exp \left(-2 G(z, \infty)-2 i G^{*}(z, \infty)\right),
$$

where $G^{*}(z, \infty)$ denotes the harmonic conjugate of $G(z, \infty)$, which is multivalued in $D$. Since $f_{1}(z)$ must be single-valued, we see that

$$
\frac{1}{2 \pi} \int_{\partial K_{1}} 2 d G^{*}=\frac{1}{\pi} \int_{\partial K_{1}} \frac{\partial G}{\partial n} d s=2 \nu\left(K_{1}\right)
$$

must be an integer. But this contradicts the assumption $\nu\left(K_{1}\right) \neq \frac{1}{2}$. 
EXAMPLE 2 [ $m=3]$. Let $0<a<b<c$.

(i) Let $K=[-c,-b] \cup[-a, a] \cup[b, c]$ be a symmetric compact set on the real line. Then $Z\left(f_{1}\right)=4$ and, hence, $c(K)=\{0\}$.

(ii) Let $K=\left\{r e^{i(2 / 3) j \pi}: a \leqslant r \leqslant b, j=0,1,2\right\}$ be a star-like compact set. Then $Z\left(f_{1}\right)=3$ but $c(K)=\{0\}$.

PROOF. (i) By symmetry and the uniqueness of the $\beta$-extremal function we easily see that $f_{1}(z)=f_{1}(-z)$ from which $Z\left(f_{1}\right)=4$ follows by using Theorem 3 .

(ii) Similarly we see $f_{1}\left(e^{i(2 / 3) \pi} z\right)=e^{i(4 / 3) \pi} f_{1}(z)$ and $Z\left(f_{1}\right)=3$. Using Lemma 4 and Theorem 5, we obtain $c(K)=\{0\}$.

EXAMPLE $3[m=4]$. Let $0<a<b<c<d$ such that $d^{2}-c^{2}=b^{2}-a^{2}$ and $K=[-d,-c] \cup[-b,-a] \cup[a, b] \cup[c, d]$. Then $c(K)=[-\delta, \delta]$ for some $\delta>0$.

Proof. Let $F_{0}$ be the Ahlfors function for the set $K^{2}=\left[a^{2}, b^{2}\right] \cup\left[c^{2}, d^{2}\right]$. As in the proof of Example 1, we see $f_{1}(z)=F_{0}\left(z^{2}\right)$ for $z \in D(K)$. Since $F$ has a simple zero at $\left(b^{2}+c^{2}\right) / 2$ by symmetry, we see that $f_{1}$ has simple zeros at $\pm \sqrt{\left(b^{2}+c^{2}\right) / 2}$. Let $G(z, \infty)$ be the Green's function of $D(K)$ with pole at $\infty$, and let

$$
P(z)=G(z, \infty)+i G^{*}(z, \infty)
$$

Then

$$
\alpha=(1 / i) P^{\prime}(z) d z
$$

is a meromorphic differential on $D(K)$ which is nonnegative along $\partial K$. Further, it is easily seen that $\alpha$ has simple zeros at $\pm \sqrt{\left(b^{2}+c^{2}\right) / 2}$ and at 0 and a simple pole at $\infty$. Therefore $\omega=z^{-1} \alpha$ satisfies the conditions of Theorem 4. Since $\omega$ has the expansion

$$
\omega=\left(i z^{-2}+\ldots\right) d z
$$

about $\infty$, we see as in the proof of Example 1 that $c(K)=[-\delta, \delta]$ for some $\delta>0$.

REMARK. Examples 1 and 3 offer a negative answer to Minsker's problem (3).

6. Concluding remarks. We do not know whether there exists any compact set $K$ with $m \neq 2,4$ for which $c(K)$ contains more than one point. In particular we know almost nothing about $c(K)$ when $K$ has infinitely many components. In this paper we answered negatively three of Minsker's problems by offering counterexamples. Minsker's fourth problem, which asks whether $c(K)$ is contained in the convex hull of $K$, is still open.

\section{REFERENCES}

1. L. Ahlfors, Bounded analytic functions, Duke Math. J. 14 (1947), 1-11. MR 9 \#24.

2. Open Riemann surfaces and extremal problems on compact subregions, Comment. Math. Helv. 24 (1950), 100-134. MR 12 \#90, 13 \#138.

3. S. Fisher, On Schwarz's lemma and inner functions, Trans. Amer. Math. Soc. 138 (1969), 229-240. MR 39 \# 1651.

4. T. Gamelin, Extremal problems in arbitrary domains, Michigan Math. J. 20 (1973), 3-11.

5. , Uniform algebra, Prentice-Hall, Englewood Cliffs, N. J., 1969.

6. Lectures on $H^{\infty}(D)$, La Plata Notas de Math., No. 21 (1972).

7. P. Garabedian, Schwarz's lemma and the Szegö kernel function, Trans. Amer. Math. Soc. 67 (1949), 1-35. MR 11 \#340.

8. D. A. Hejhal, Linear extremal problems for analytic functions, Acta Math. 128 (1972), 91-122.

9. S. Minsker, Analytic centers and analytic diameters of planar continua, Trans. Amer. Math. Soc. 191 (1974), 83-93. 
10. Ch. Pommerenke, Uber die analytische Kapazitat, Arch. Math. 11 (1960), 270-277.

11. W. Rudin, Analytic functions of class $H_{p}$, Trans. Amer. Math. Soc. 78 (1955), 46-66.

12. __ Real and complex analysis, 2nd ed., McGraw-Hill, New York, 1974.

13. A. G. Vitushkin, Necessary and sufficient conditions on a set in order that any continuous function analytic at the interior points of the set may admit uniform approximation by rational functions, Dokl. Akad. Nauk SSSR 171 (1966), 1255-1258 = Soviet Math. Dokl. 7 (1966), 1622-1625. MR 35 \# 391.

14. Analytic capacity of sets and problems in approximation theory, Uspehi Mat. Nauk 22 (1967), no. 6 (138), 141-199 = Russian Math. Surveys 22 (1967), no. 6, 139-200. MR 37 \# 5404.

Department of Mathematics, Tokyo Institute of Technology, Oh-Okayama, Meguro-Ku, TOKYO, JAPAN (Current address of Nobuyuki Suita)

Current address (Shōji Kobayashi): Department of Electric Engineering, Technological University of Nagaoka, Nagaoka, Niigata, Japan 